Reprod. Nutr. Dévelop., 1987, 27 (4), 755-768.

\title{
Nature de l'aliment, finesse de mouture et données anatomo-fonctionnelles du tube digestif proximal du lapin
}

\author{
A. AUVERGNE, Th. BOUYSSOU $(*)$, M. PAIRET $(*)$, M. BOUILLIER-OUDOT, \\ Y. RUCKEBUSCH $\left({ }^{*}\right)$, M. CANDAU
}

Service des Productions Animales, Ecole Nationale Supérieure Agronomique, 145, avenue de Muret, 31076 Toulouse Cedex, France.

(*) Laboratoire de Physiologie, Ecole Nationale Vétérinaire, 23, chemin des Capelles, 31076 Toulouse Cedex, France.

Summary. Diet type, grinding size and anatomo-functional characteristics of the proximal digestive tract in rabbits.

Two diets containing either dehydrated lucerne $(40 \%)$ or dehydrated beet pulp $(50 \%)$ both being coarsely $(4 \mathrm{~mm})$ of finely $(1 \mathrm{~mm})$ ground before pelleting, were fed to 120 rabbits after weaning. Feed intake and weight gain were estimated.

After 79 days, 11 rabbits in each group were slaughtered between 14.00 and $17.00 \mathrm{~h}$. The parameters measured were stomach and ileal weights, dry matter, fibre and nitrogen contents, volatile fatty acid concentrations. Gastric emptying and intestinal transit time were estimated in each group.

Rabbits fed the beet pulp diet had a better feed conversion ratio associated with a higher solid matter gastric retention and also a longer jejuno-ileal transit time.

Finely ground diet further increased the transit time, particularly in the ileon, which was also associated with a higher concentration of fermentation by-products.

\section{Introduction.}

L'influence de la nature des glucides pariétaux ou de la finesse de mouture de l'aliment sur le fonctionnement digestif chez le Lapin a fait l'objet de divers travaux. Ces derniers mettent en évidence une influence de la source de cellulose (Colin et al., 1976 ; Lebas et Laplace, 1977) et de la taille des particules de I'aliment (Laplace et Lebas, 1977 ; Morisse, 1982) sur le transit digestif.

La granulométrie d'un aliment étant dépendante des matières premières et de la finesse de mouture de cet aliment, il semble intéressant d'envisager les effets de ces deux paramètres (matières premières et finesse de mouture) sur le transit gastro-intestinal (de la bouche à la valvule iléo-caecale) et sur l'importance et la nature des fermentations dans ces segments. 


\section{Matériel et méthodes.}

1) Dispositif expérimental. - Deux aliments complets, différant par la nature de leurs glucides membranaires, l'un à base de luzerne déshydratée, l'autre à base de pulpe de betterave déshydratée, sont présentés sous forme de granulés $15 \mathrm{~mm}$ de diamètre) à des lapereaux à partir du sevrage (30 jours). Pour chaque aliment, dénommé par la suite aliment pulpe ou aliment luzerne, deux finesses de mouture, caractérisées par le diamètre de perforation de la grille de broyeur : $1 \mathrm{~mm}$ ou $4 \mathrm{~mm}$, sont utilisées. La composition centésimale de ces rations est identique à celles décrites par Pairet et al. (1986). Leur composition chimique est portée au tableau 1.

Le profil granulométrique des aliments est réalisé par voie humide selon la méthode de Lebas (communication personnelle), décrite par Pairet et al. (1986).

TABLEAU 1

Composition chimique des aliments expérimentaux (exprimée en \% de la matière sèche).

\begin{tabular}{|c|c|c|c|c|}
\hline \multirow{2}{*}{$\begin{array}{l}\text { Régime } \\
\text { Finesse de mouture }\end{array}$} & \multicolumn{2}{|c|}{ Luzerne } & \multicolumn{2}{|c|}{ Pulpe } \\
\hline & $1 \underset{\text { (L1) }}{\mathrm{mm}}$ & $\underset{\text { (L4) }}{4 \mathrm{~mm}}$ & $1 \underset{(P 1)}{\mathrm{mm}}$ & $\begin{array}{c}4 \mathrm{~mm} \\
(\mathrm{P} 4)\end{array}$ \\
\hline \multicolumn{5}{|l|}{ Critères : } \\
\hline \multicolumn{5}{|l|}{ Analyse fourragère } \\
\hline $\begin{array}{l}\text { Matière sèche } \\
\text { Matières azotées totales } \\
\text { Cellulose brute } \\
\text { Matières grasses } \\
\text { Matières minérales } \\
\text { Energie brute }\left(^{*}\right) \text { (kcal/kg aliment) }\end{array}$ & $\begin{array}{r}89,5 \\
16,1 \\
14,9 \\
1,9 \\
9,2 \\
4230\end{array}$ & $\begin{array}{r}88,2 \\
16,2 \\
16,3 \\
2,0 \\
9,1 \\
4250\end{array}$ & $\begin{array}{r}90,8 \\
16,6 \\
12,3 \\
1,4 \\
5,4 \\
4350\end{array}$ & $\begin{array}{r}90,1 \\
16,1 \\
13,1 \\
1,4 \\
5,3 \\
4350\end{array}$ \\
\hline \multicolumn{5}{|l|}{ Composition en glucides pariétaux } \\
\hline $\begin{array}{l}\text { Lignocellulose (Acide Detergent Fiber) } \\
\text { Neutral Detergent Fiber } \\
\text { Cellulose (Van Soest) } \\
\text { Hémicellulose (Van Soest) } \\
\text { Lignine }\end{array}$ & $\begin{array}{r}18,5 \\
30,6 \\
13,0 \\
12,1 \\
5,5\end{array}$ & $\begin{array}{r}20,4 \\
34,2 \\
14,3 \\
13,7 \\
6,1\end{array}$ & $\begin{array}{r}17,2 \\
37,2 \\
13,7 \\
20,1 \\
3,4\end{array}$ & $\begin{array}{r}17,8 \\
38,1 \\
13,6 \\
20,4 \\
4,3\end{array}$ \\
\hline
\end{tabular}

(*) Energie brute, calculée selon l'équation de Schiemann (1971).

2) Etude des contenus. - Cent vingt lapins de race néo-zélandaise, des deux sexes, sont répartis dès le sevrage en cage collective de trois animaux, sexes mélangés, selon la méthode des blocs décrite par Morin et al. (1979). Ils reçoivent à volonté l'un des quatre aliments expérimentaux (pulpe 1, pulpe 4, luzerne 1, luzerne 4) pendant la période de croissance (30-79 jours).

Les performances zootechniques des animaux sont appréciées au cours de l'essai par mesure de la croissance et du niveau d'ingestion. La croissance est estimée par pesée à $30,37,51,58,72,79$ jours. Le niveau d'ingestion est calculé deux fois par semaine à partir de la pesée des quantités distribuées et des refus. 
Le développement pondéral des différents viscères et certaines caractéristiques de leurs contenus sont estimés par abattage à 79 jours de 11 lapins choisis de manière aléatoire dans chaque lot. Après insensibilisation par dislocation cervicale, les animaux sont saignés, dépouillés et éviscérés, entre $14 \mathrm{~h}$ et $17 \mathrm{~h}$. Les poids de l'estomac et de l'iléon (portion intestinale comprise entre l'avant-dernière anse mésentérique et la valvule iléocaecale exclue) sont déterminés par différence de pesée des organes pleins et vides laprès lavage et ressuyage au papier Joseph). Le contenu des différents réservoirs digestifs est collecté dans des pots, en présence de chlorure mercurique afin d'arrêter les fermentations.

Les paramètres suivants ont été retenus pour caractériser la composition du contenu de ces organes : teneurs en matière sèche, en azote, en composants pariétaux et en acides gras volatils.

La détermination de la matière sèche est effectuée par passage à l'étuve à $60{ }^{\circ} \mathrm{C}$ et pesée à poids constant.

Le fractionnement des constituants pariétaux est réalisé par les méthodes de Van Soest (1967) (Acid detergent fiber, Neutral detergent fiber, méthode de dosage de la lignine au permanganate) sur $1 \mathrm{~g}$ de contenu frais, ce qui permet d'évaluer les taux d'hémicellulose (NDF - ADF) et la cellulose (ADF - lignine).

L'azote total est mesuré par la méthode de Kjeldahl, les acides gras volatils par chromatographie en phase gazeuse sur un chromatographe Intersmat IGC 120 FB (phase stationnaire : $10 \%$ SP 1 200/I \% $\mathrm{H}_{3} \mathrm{PO}_{4}$ (Supelco) - support chromosorb W.AW 82/100), avec l'acide valérique comme étalon interne.

3) Etude du transit. - Le temps de transit entre la bouche et l'iléon est mesuré par la technique de dilution d'un traceur. Quatre lapins, munis d'une fistule iléale (diamètre $4 \mathrm{~mm}$ ) placée à $5 \mathrm{~cm}$ de la valvule iléocaecale, reçoivent successivement pendant trois semaines les quatre régimes, suivant un plan expérimental en carré latin, à partir de l'âge de 7 semaines.

Après deux semaines d'accoutumance, une dose de phénol-sulfone-phtaléine (PSP) $(1 \mathrm{ml}$; solution à $25 \mathrm{mg} / \mathrm{ml}$ ) est administrée par voie orale (sonde œsophagienne). Les contenus iléaux sont ensuite recueillis toutes les $5 \mathrm{~min}$ pendant 3 heures. Leur concentration en PSP est mesurée par colorimétrie $(523 \mathrm{~nm})$ après dilution, défécation et alcalinisation par $\mathrm{Na}_{3} \mathrm{PO}_{4}$ (Barreiro, McKenna et Beck, 1968). Le temps de transit est apprécié par le délai qui sépare l'ingestion de PSP et le pic de sa concentration dans les contenus.

La vidange gastrique est étudiée par la technique de dilution du polyéthylène glycol 4000 (PEG 4000) sur un groupe de huit lapins âgés de 7 semaines, équipés d'une canule en pyrex $(10 \mathrm{~mm}$ de diamètre) sur le fundus, selon le plan expérimental décrit pour la mesure du transit bouche-iléon.

Cinq $\mathrm{ml}$ d'une solution aqueuse contenant $0,5 \mathrm{~g}$ de traceur sont induits par la canule. Des prélèvements de contenu sont effectués toutes les 30 min pendant les trois premières heures et toutes les $60 \mathrm{~min}$ les deux heures suivantes.

Après dilution et défécation, le PEG est dosé selon la méthode turbidimétrique de Hyden (1955).

Afin d'empêcher la caecotrophie et la prise de nourriture, les lapins sont placés en cage à contention pendant la durée des manipulations. Toutes les expériences débutent à $14 \mathrm{~h}$. 


\section{Résultats.}

1) Les aliments expérimentaux. - Les aliments expérimentaux sont isoazotés (tabl. 1) et isocellulosiques (cellulose Van Soest). Ils diffèrent par leurs teneurs en hémicellulose (plus élevée dans l'aliment pulpe) et en lignine (plus élevée dans l'aliment luzerne).

On peut noter, pour le régime luzerne, des différences sensibles des teneurs analytiques en constituants pariétaux en fonction de la finesse de mouture. La diminution de la taille des particules s'accompagne d'une baisse apparente de la teneur en ces constituants. Dans la mesure où la fabrication des deux aliments expérimentaux de ce régime luzerne est effectuée à partir du même mélange de matières premières, les différences observées sont imputables aux méthodes de dosage et en particulier à des pertes de matière au moment des filtrations pour les substrats finement broyés.

Les régimes moulus grossièrement (L4 et P4) présentent (tabl. 2) les taux de particules de tailles supérieures à $1 \mathrm{~mm}$ les plus élevés $(13,1 \%$ et $16,2 \%$ vs $4,5 \%$ et $11,1 \%$ ). Les rations à base de luzerne ont des taux de particules solubles et fines nettement plus importants que les rations à base de pulpe, ce qui est la conséquence des traitements technologiques différents subis par ces matières premières. Pour les autres plages granulométriques, le délitage en milieu humide favorise l'agrégation des particules des aliments à base de pulpe dont le pourcentage en particules grossières est ainsi surestimé.

TABLEAU 2

Profil granulométrique (en \% de MS).

\begin{tabular}{|c|c|c|c|c|c|c|}
\hline & \multicolumn{5}{|c|}{ Taille des particules $(\mathrm{mm})$} & \multirow{2}{*}{$\begin{array}{l}\text { Solubles } \\
\text { et fines }\end{array}$} \\
\hline & $>1$ & $>0,5$ & $>0,315$ & $>0,100$ & $>0,05$ & \\
\hline $\begin{array}{l}\text { L1 } \\
\text { L4 } \\
\text { P1 } \\
\text { P4 }\end{array}$ & $\begin{array}{r}4,5 \\
13,1 \\
11,1 \\
16,2\end{array}$ & $\begin{array}{l}15,6 \\
13,8 \\
20,2 \\
18,7\end{array}$ & $\begin{array}{r}11,9 \\
9,8 \\
12,3 \\
11,0\end{array}$ & $\begin{array}{l}15,0 \\
14,4 \\
13,9 \\
12,1\end{array}$ & $\begin{array}{l}5,4 \\
5,0 \\
5,2 \\
3,4\end{array}$ & $\begin{array}{l}47,5 \\
43,9 \\
37,4 \\
36,7\end{array}$ \\
\hline
\end{tabular}

2) Paramètres zootechniques. - Sur l'ensemble de la période expérimentale, les résultats, reportés dans le tableau 3 , indiquent que le régime et la finesse de mouture n'exercent pas d'influence significative sur la croissance.

Le niveau d'ingestion des animaux recevant l'aliment luzerne est, par contre, significativement plus élevé $(P<0,01)$ que celui des animaux du lot pulpe à partir de l'âge de 51 jours, mais l'efficacité alimentaire est plus faible (écart moyen sur la période expérimentale entre les indices de consommation $=0,4$ ). Quelle que soit la nature de l'aliment expérimental, une mouture plus fine conduit $(P<0,10)$ à une baisse du niveau d'ingestion et à une amélioration significative $(P<0,05)$ de l'indice de consommation. La meilleure efficacité alimentaire de l'aliment pulpe se manifeste très rapidement dès la seconde semaine d'ingestion de l'aliment expéri- 


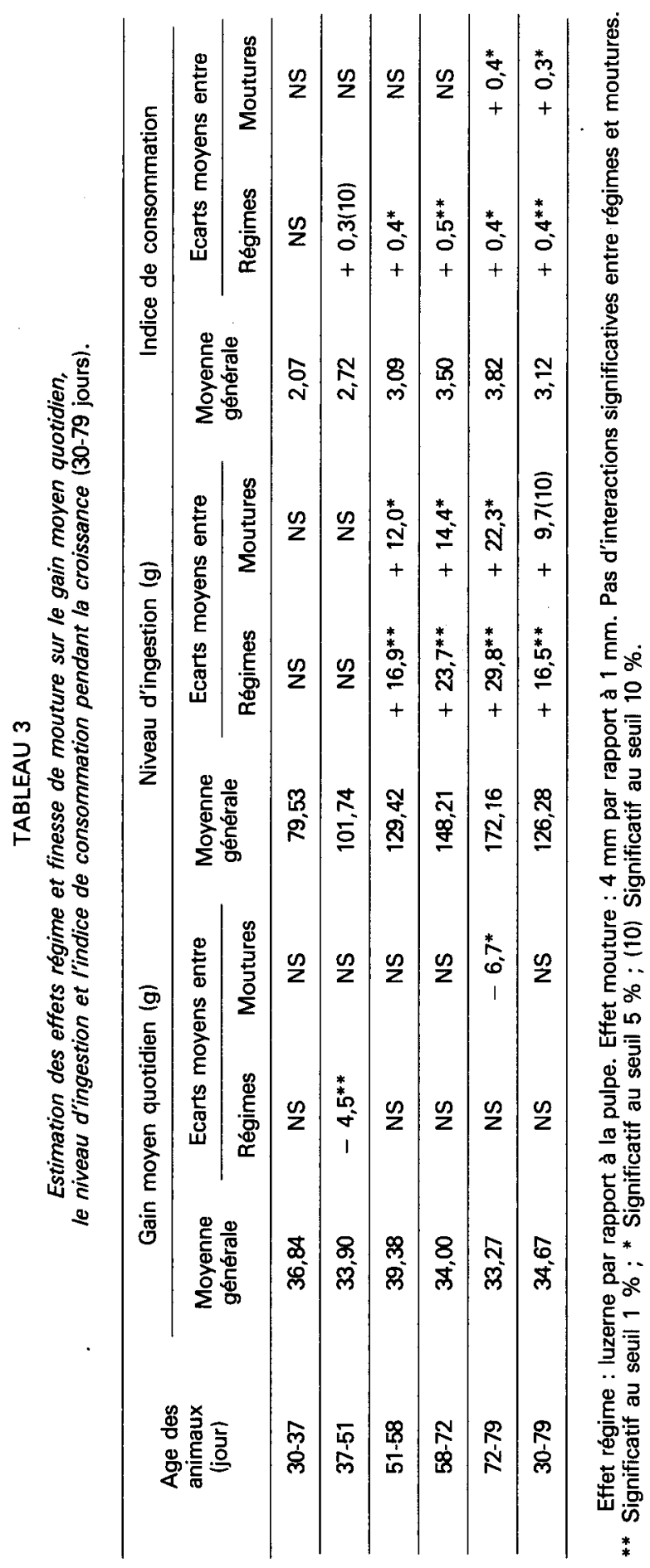


mental. L'influence de la finesse de mouture s'exerce significativement $(P<0,05)$ sur le niveau d'ingestion vers la quatrième semaine (baisse du niveau d'ingestion pour les animaux ingérant une mouture fine) et sur l'indice de consommation en septième semaine d'expérience.

Malgré l'absence de différence significative, l'ingestion de l'aliment pulpe s'est traduite par un taux de mortalité par diarrhée relativement élevé par rapport à la consommation de l'aliment luzerne (15 morts/60 animaux vs 9 morts/60 animaux). Les moutures fines $(1 \mathrm{~mm})$ ont un effet similaire (16 morts/60 animaux vs 8 morts/60 animaux pour les moutures grossières $(4 \mathrm{~mm}))$. Par contre, quelques cas de mortalité par parésie caecale ont été observés dans le lot des sujets ingérant les aliments à mouture $4 \mathrm{~mm}$ (3 morts pour le lot L4, 1 mort pour le lot P4).

3) Composition des contenus gastriques. - Les animaux, au moment de l'abattage entre $14 \mathrm{~h}$ et $17 \mathrm{~h}$, se trouvent en phase finale de caecotrophie, ce que suggèrent les fortes teneurs en azote total des contenus gastriques. Les poids des contenus frais de l'estomac (tabl. 4) sont plus faibles chez les animaux alimentés à base de luzerne $(-15 \mathrm{~g} \mathrm{P}<0,05)$, le taux de matière sèche du contenu est plus élevé pour ces animaux $(+2,17 \%<0,01)$. La finesse de mouture est sans effet sur le contenu stomacal chez les animaux nourris à base de luzerne alors qu'une mouture fine associée à la pulpe diminue les contenus et les taux de matières sèches (interaction significative).

TABLEAU 4

Poids et composition du contenu gastrique (matière sèche, azote, constituants pariétaux et acides gras volatils (AGV) p. $100 \mathrm{~g}$ de matière sèche).

\begin{tabular}{|c|c|c|c|c|c|c|c|}
\hline \multirow[b]{2}{*}{ Critères } & \multicolumn{2}{|c|}{ Luzerne } & \multicolumn{2}{|c|}{ Pulpe } & \multicolumn{2}{|c|}{ Ecart-moyen } & \multirow[b]{2}{*}{$\begin{array}{l}\text { Inter- } \\
\text { action }\end{array}$} \\
\hline & $\begin{array}{c}1 \mathrm{~mm} \\
(\mathrm{n}=11)\end{array}$ & $\begin{array}{c}4 \mathrm{~mm} \\
(\mathrm{n}=11)\end{array}$ & $\begin{array}{c}1 \mathrm{~mm} \\
(\mathrm{n}=11)\end{array}$ & $\begin{array}{c}4 \mathrm{~mm} \\
(\mathrm{n}=11)\end{array}$ & $\begin{array}{c}\text { Entre } \\
\text { régimes } \\
\text { (L-P) }\end{array}$ & $\begin{array}{c}\text { Entre } \\
\text { moutures } \\
(4-1)\end{array}$ & \\
\hline $\begin{array}{l}\text { Poids du contenu frais } \\
\text { (g/kg PV) }\end{array}$ & 28,2 & 29,9 & 42,9 & 45,8 & $-15,3^{*}$ & NS & NS \\
\hline $\mathrm{pH}$ & 1,14 & 1,15 & 1,21 & 1,39 & NS & NS & NS \\
\hline \% Matière sèche & 17,92 & 15,58 & 13,16 & 16,01 & $+2,17^{* *}$ & NS & $* *$ \\
\hline Azote total $(\mathrm{g})$ & 2,73 & 3,66 & 3,79 & 4,75 & $-1,07^{*}$ & $+0,94^{*}$ & NS \\
\hline Hémicellulose (g) & 20,7 & 21,7 & 15,2 & 18,5 & $+4,3^{* *}$ & NS & NS \\
\hline Cellulose $(\mathrm{g})$ & 22,5 & 24,4 & 16,0 & 17,1 & $+7,9^{* *}$ & NS & NS \\
\hline Lignine $(g)$ & 8,0 & 9,8 & 3,3 & 4,8 & $+4,8^{* *}$ & NS & NS \\
\hline AGV totaux (mM) & 6,71 & 5,42 & 5,73 & 5,34 & NS & NS & NS \\
\hline Acide acétique (mM) & 6,71 & 5,42 & 5,73 & 5,34 & NS & NS & NS \\
\hline
\end{tabular}

Effet régime : valeur luzerne moins valeur relative à la pulpe. Effet mouture : valeur « $4 \mathrm{~mm}$ » moins valeur « $1 \mathrm{~mm}$ ».

$\mathbf{n}=$ effectif ; ** Significatif au seuil $1 \%$; * Significatif au seuil $5 \%$. 
La teneur en azote du contenu gastrique est significativement influencée par le régime $13,20 \%$ d'azote total par rapport à la matière sèche pour le régime luzerne versus $4,27 \%$ pour le régime pulpe) et par la finesse de mouture $(3,26 \%$ d'azote total par rapport à la matière sèche pour la mouture fine versus $4,20 \%$ pour la mouture grossière).

Les teneurs des contenus en constituants pariétaux (hémicellulose, cellulose et lignine) sont très significativement plus élevées chez les animaux recevant de la luzerne (respectivement $+4,3 \%,+7,9 \%$ et $+4,8 \%$ ). L'ingestion des aliments à mouture fine se traduit par une légère diminution de ces teneurs sans que l'effet soit significatif. Aucun effet significatif des traitements (aliment ou mouture) n'est enregistré sur les concentrations des contenus en acides gras volatils, ou en acide acétique (seul acide détecté dans les contenus).

4) Composition des contenus iléaux. - Au niveau de l'iléon (tabl. 5), l'ingestion de la luzerne ou d'un aliment à mouture grossière se traduit par des poids de

TABLEAU 5

Poids et composition du contenu iléal (matière sèche, azote, constituants pariétaux et acides gras volatils (AGV) p. $100 \mathrm{~g}$ de matière sèche).

\begin{tabular}{|c|c|c|c|c|c|c|c|}
\hline \multirow{2}{*}{ Critères } & \multicolumn{2}{|c|}{ Luzerne } & \multicolumn{2}{|c|}{ Pulpe } & \multicolumn{2}{|c|}{ Ecart-moyen } & \multirow[b]{2}{*}{$\begin{array}{l}\text { Inter- } \\
\text { action }\end{array}$} \\
\hline & $\begin{array}{c}1 \mathrm{~mm} \\
(\mathrm{n}=11)\end{array}$ & $\begin{array}{c}4 \mathrm{~mm} \\
\langle\mathrm{n}=11\rangle\end{array}$ & $\begin{array}{c}1 \mathrm{~mm} \\
(\mathrm{n}=11)\end{array}$ & $\begin{array}{c}4 \mathrm{~mm} \\
(\mathrm{n}=11)\end{array}$ & $\begin{array}{c}\text { Entre } \\
\text { régimes } \\
\text { (L-P) }\end{array}$ & $\begin{array}{c}\text { Entre } \\
\text { moutures } \\
(4-1)\end{array}$ & \\
\hline $\begin{array}{l}\text { Poids du contenu frais } \\
(\mathrm{g} / \mathrm{cm})\end{array}$ & 0,152 & 0,130 & 0,194 & 0,164 & $-0,068^{*}$ & $-0,044^{*}$ & NS \\
\hline $\mathrm{pH}$ & 7,60 & 7,77 & 7,59 & 7,66 & NS & NS & NS \\
\hline \% Matière sèche & 11,21 & 11,99 & 11,99 & 10,91 & NS & NS & NS \\
\hline Azote total $(\mathrm{g})$ & 2,90 & 3,02 & 2,92 & 3,10 & NS & NS & NS \\
\hline Hémicellulose $(\mathbf{g})$ & 14,7 & 17,6 & 14,9 & 19,1 & NS & $+3,5^{* *}$ & NS \\
\hline Cellulose $(g)$ & 16,4 & 22,2 & 16,7 & 19,9 & NS & $+4,6^{* *}$ & NS \\
\hline Lignine $(g)$ & 5,8 & 8,7 & 4,9 & 6,6 & $+1,5^{* *}$ & $+2,3^{* *}$ & NS \\
\hline$A G V$ totaux $(\mathrm{mM})$ & 17,19 & 11,91 & 14,66 & 12,06 & NS & $-3,95^{* *}$ & NS \\
\hline Acide acétique (mM) & 11,83 & 5,20 & 7,03 & 6,94 & $+1,53(10)$ & $-3,36^{* *}$ & $* *$ \\
\hline Acide propionique (mM) & 0,57 & 0,53 & 0,49 & 0,39 & NS & NS & NS \\
\hline Acide isobutyrique (mM) & 1,26 & 2,89 & 3,22 & 1,79 & NS & NS & ** \\
\hline Acide butyrique (mM) & 2,11 & 2,30 & 1,41 & 1,43 & $+0,78^{*}$ & NS & NS \\
\hline Acide isovalérique (mM) & 1,49 & $1, \infty$ & 2,49 & 1,50 & $+0,75^{* *}$ & $-0,74^{*}$ & NS \\
\hline
\end{tabular}

Effet régime : valeur luzerne moins valeur relative à la pulpe. Effet mouture : valeur « $4 \mathrm{~mm}$ » moins valeur " $1 \mathrm{~mm}$ ». $10 \%$.

$n=$ effectif. ${ }^{*}$ Significatif au seuil $1 \%$. * Significatif au seuil $5 \%$. (10) Significatif au seuil de 
contenus frais plus faibles $(-0,068 \mathrm{~g} / \mathrm{cm}$ et $-0,044 \mathrm{~g} / \mathrm{cm}$ de contenu frais respectivement) sans modifier significativement les taux de matière sèche $(11,53 \%$ en moyenne).

L'influence du régime sur la composition en constituants pariétaux de ce contenu s'exerce principalement et significativement au niveau de la teneur en lignine qui est accrue dans le cas de l'aliment luzerne $(+1,5 \%$ MS P $<0,01)$.

L'influence de la finesse de mouture est marquée pour l'ensemble de ces paramètres : les teneurs en hémicellulose, cellulose et en lignine des contenus iléaux des animaux recevant les moutures grossières sont significativement plus importantes (respectivement $+3,5 \%,+4,6 \%,+2,3 \% P<0,01$ ).

De façon générale, les concentrations en acides gras volatils totaux et en acides isobutyrique et isovalérique des contenus iléaux sont particulièrement élevées. Le régime a des effets significatifs et opposés sur les teneurs en acides butyrique et isovalérique, une teneur élevée en acide butyrique $(+0,78 \% \mathrm{MS} P<0,05)$ et faible en acide isovalérique $(-0,75 \% \mathrm{MS} P<0,01)$ étant associée au régime luzerne. La finesse de mouture exerce un effet significatif au niveau des concentrations en acides gras volatils totaux, en acides acétique et isovalérique qui sont abaissées par les moutures grossières.

Les interactions observées mettent en évidence des effets différents des matières premières selon la finesse de mouture qui leur est appliquée. L'ingestion de luzerne grossière déprime fortement la concentration en acide acétique du contenu alors que celle des pulpes $4 \mathrm{~mm}$ l'augmente. La situation inverse s'observe dans le cas de l'acide isobutyrique.

5) Vidange gastrique et transit bouche-iléon. - La vitesse d'élimination gastrique du PEG 4000, marqueur liquide, est identique quel que soit le régime (fig. 1). Par contre, le transit de la bouche à la valvule iléocaecale (fig. 2) de la phénol-sulfone-phtaléine (PSP), autre marqueur de la phase liquide, est nettement plus rapide chez les animaux recevant des régimes luzerne : une heure après l'ingestion, on recueille 7 points de PSP de plus, le délai d'apparition du pic de concentration est significativement diminué $(-29 \min P<0,01)$. L'effet de la finesse de mouture est également significatif bien que plus faible que celui de la nature du régime : les moutures grossières accélèrent le transit du marqueur liquide $(+4,5$ points une heure après l'ingestion) $(P<0,001)$ et le délai d'apparition du pic de concentration est diminué de 7 min $(P<0,10)$.

\section{Discussion et conclusions.}

1) Influence du régime. - L'influence du régime s'exerce d'une part sur l'efficacité alimentaire qui est nettement accrue pour les animaux ingérant l'aliment pulpe, d'autre part sur le poids des contenus gastriques et iléaux plus élevés avec ce type d'aliment. Ces deux types de paramètres ne sont pas indépendants. En effet, le meilleur indice de consommation enregistré avec l'aliment pulpe par rapport à l'aliment luzerne peut être relié à une utilisation digestive accrue (Candau et al., 1978b) du fait de la différence de composition en hémicellulose et en 


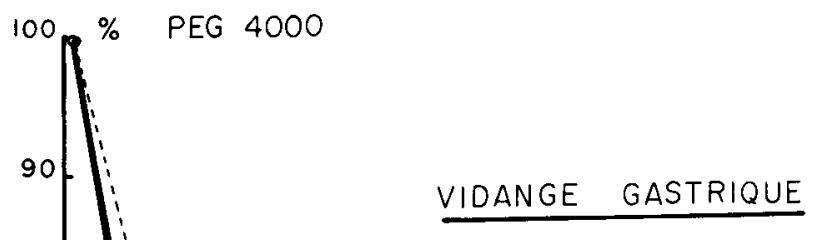

FIG. 1. - Vidange gastrique ${ }^{(1)}: \%$ PEG $=100 \times T^{b}(\log (\%$ PEG $)=a+b(\log (T))$.

\begin{tabular}{|c|c|c|c|}
\hline \multirow{2}{*}{$\begin{array}{c}\text { Constante } \\
\text { d'élimination } \\
\text { du PEG } \\
\text { (coefficient b) }\end{array}$} & \multicolumn{2}{|c|}{ Signification statistique } & Test $F$ \\
\hline & $\begin{array}{l}\text { Linéarité } \\
\text { (F) }\end{array}$ & Parallélisme & $\begin{array}{c}\text { Régression } \\
\text { moyenne }\end{array}$ \\
\hline$-0,691$ & $1,06 \mathrm{NS}$ & $0,21 \mathrm{NS}$ & $477,46^{* * *}$ \\
\hline
\end{tabular}

(1) Les trois premières heures suivant l'administration.

*** Signification à $\mathrm{P}<0,001$.

$T$ : temps en minutes. 


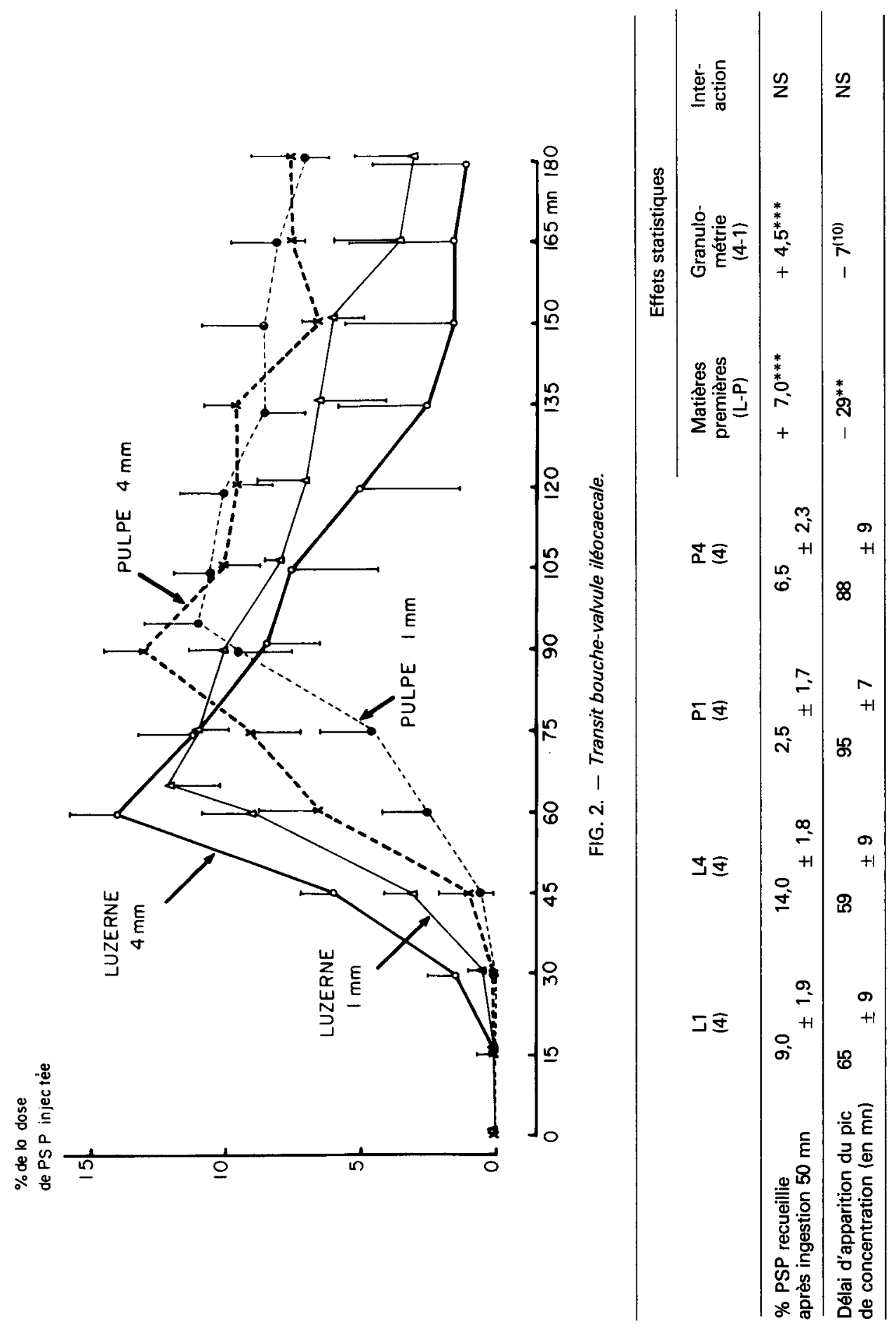


lignine des matières premières et du ralentissement du transit digestif observé avec I'aliment pulpe (Laplace et Lebas, 1977).

Au niveau gastrique, le contenu est représenté par une phase liquide et une phase solide (mélange d'aliments et de caecotrophes). La vidange du marqueur de la phase liquide est identique quel que soit le régime et plus rapide que celle de la phase solide (Pickard et Stevens, 1972). L'augmentation du contenu sec de l'estomac des lapins ingérant le régime pulpe pourrait être due à un accroissement de la proportion de caecotrophes présents dans le mélange. En effet, Dehalle (1981) constate que la production quantitative de caecotrophes n'est pas modifiée sur $24 \mathrm{~h}$ par la concentration énergétique, alors que l'ingestion d'aliment est réduite. De ce fait, il est normal de supposer qu'entre $14 \mathrm{H}$ et $17 \mathrm{~h}$ le contenu gastrique des animaux ingérant l'aliment le plus énergétique, c'est-à-dire l'aliment pulpe, est proportionnellement plus riche en caecotrophes. Ceci est confirmé par un taux d'azote total plus élevé dans le contenu gastrique des animaux du lot pulpe.

Les modifications de proportion dans les composants du mélange gastrique sont susceptibles d'expliquer l'augmentation du contenu dans l'estomac de ces animaux, Gidenne et Poncet (1985) montrent en effet que les caecotrophes sont retenus plus longtemps que les aliments dans l'estomac. Globalement, dans le cas d'un régime à base de pulpe, la vidange du contenu sec est donc ralentie, du moins pendant la période étudiée, ce qui est à relier avec la mauvaise coordination auto-duodénale enregistrée avec ce type de régime (Pairet et al., 1986).

Pour le régime pulpe, ce ralentissement de la vidange gastrique de la phase solide, alors que celle de la phase solide n'est pas modifiée, suggère un contenu quittant l'estomac plus dilué. Or, les mesures du transit intestinal montrent un ralentissement global de la phase liquide (décalage de $29 \mathrm{~min}$ du pic de concentration en phénol-sulfone-phtaléine au niveau de la valvule iléocaecale) avec le régime pulpe. Si la migration de la phase solide le long de l'intestin n'était pas influencée par le régime, on devrait donc enregistrer au niveau iléal d'une part une diminution de la teneur en matière sèche du contenu et d'autre part une arrivée de matière sèche réduite. Or, inversement, on observe des taux de matière sèche non spécifiquement différents entre régimes et par contre un accroissement de $30 \%$ environ du contenu iléal. Ceci suppose donc un ralentissement du transit intestinal pour la phase solide que l'on peut relier à l'effet également très significatif de ce régime sur la motricité jéjuno-iléale : Pairet et al. (1986) observent pratiquement une diminution de moitié de l'occurrence des complexes myoélectriques migrants à ce niveau avec le régime pulpe. De plus, la comparaison des teneurs en lignocellulose des contenus gastriques et iléaux des lots pulpe et luzerne montre que, par rapport au contenu gastrique, le contenu iléal s'appauvrit $(-18 \%)$ en lignocellulose dans le cas des régimes luzerne et au contraire s'enrichit $(+17 \%)$ en ces constituants dans le cas des régimes pulpe bien que les fermentations (AGV totaux) soient équivalentes. Ceci corrobore donc la stase iléale du contenu intestinal des animaux ingérant un régime à base de pulpe.

L'origine de cet effet reste à préciser. En effet, si certains auteurs (Svendsen, 1972 ; Vigroux, 1976) ont noté une sensibilité de la paroi intestinale aux acides gras volatils, cet aspect ne peut être retenu pour l'effet de l'aliment pulpe dont 
l'ingestion ne se traduit aucunement par rapport à l'aliment luzerne par des modifications significatives de concentration en acides gras volatils tant au niveau gastrique qu'iléal. Quelle que soit l'étiologie de cette hypomobilité intestinale, le fait qu'elle se traduise par une augmentation des troubles diarrhéiques chez les animaux ingérant l'aliment pulpe est en accord avec la plupart des résultats bibliographiques (Laplace, 1978).

Nos résultats montrent par ailleurs l'existence d'une fermentation iléale non négligeable. Les concentrations en acides gras volatils atteignent en effet des valeurs notables (11 à $17 \mathrm{mM} / 100 \mathrm{~g} \mathrm{MS}$ ) contrairement aux résultats de Vernay (1985). Ces différences sont peut-être imputables aux conditions expérimentales. Les contenus obtenus par abattage des animaux entre $14 \mathrm{~h}$ et $17 \mathrm{~h}$ présentent des concentrations en acides gras volatils maximales, correspondant à l'arrivée d'un mélange d'aliments et de caecotrophes dégradés dans l'estomac.

2) Influence de la finesse de mouture. - Les moutures fines $(1 \mathrm{~mm})$ des aliments réduisent le niveau d'ingestion et améliorent l'indice de consommation dans la seconde partie de l'engraissement. Ceci est en accord avec les résultats de Laplace et Lebas (1977).

Contrairement à l'effet du régime, la mouture n'a pas d'incidence significative sur les paramètres du contenu gastrique sauf au niveau de la teneur en azote : taux d'azote total de la matière sèche plus faible. Pour des poids de contenu gastrique équivalents, ceci suggère une augmentation de la proportion d'aliments dans le mélange aliments (mouture fine) et caecotrophes. De même, l'évacuation gastrique de la phase liquide des digesta n'apparaît pas être modifiée de façon appréciable par la mouture. Par contre, l'effet mouture est significatif sur le transit intestinal de la phase liquide des digesta qui est nettement ralenti pour les régimes à fine mouture. Pour ces régimes, l'augmentation du poids du contenu sec iléal permet d'émettre l'hypothèse d'un ralentissement parallèle de la phase solide. L'enregistrement de la motricité jéjuno-iléocaecale confirme cette hypothèse : activité rapide abaissée et médiocre coordination iléocaecale (Pairet et al., 1986) avec les régimes à fine mouture. Ce ralentissement s'accompagne de fermentations iléales significativement plus intenses dans le cas des régimes à fine mouture : accroissement de $28 \%$ en moyenne des concentrations en acides gras volatils totaux correspondant à une dégradation accrue des constituants pariétaux (teneurs significativement plus faibles en cellulose et hémicellulose des constituants secs iléaux) qui pourrait également avoir pour origine une augmentation des surfaces d'attaque pour les microorganismes présents.

Les faciès microbiens en cause manifestent a priori des différences entre les aliments pulpe et luzerne. En effet, l'augmentation de l'acidité volatile totale enregistrée avec l'augmentation de la finesse de broyage de l'aliment luzerne est essentiellement due à un accroissement de la concentration en acide acétique $(+128 \%)$, alors que dans le cas de l'aliment pulpe ce sont les teneurs en acides isobutyrique et isovalérique qui sont accrues $1+80 \%$ et $+66 \%$ respectivement).

On peut remarquer que le lot des animaux ingérant l'aliment pulpe mouture fine c'est-à-dire celui dont le transit intestinal est le plus ralenti, présente le taux de mortalité par diarrhée le plus élevé. 
En résumé, nature du régime et finesse de mouture ont des effets nets sur l'efficacité alimentaire qui peuvent être rapprochés de modifications sensibles du transit dans le tube digestif proximal et des fermentations dans l'iléon. Des études en cours visent à préciser les mécanismes en cause dans les modifications de motricité que nous avons pu relier aux variations de transit induites par ces différents régimes.

Reçu en janvier 1985.

Accepté en mars 1987.

\section{Références}

BARREIRO M. A., Mc KENNA R. D., BECK I. I., 1968. Delimitation of transit time in the human jejunum in the simple injection indicator-dilution technique. Ann. J. Dig. Dis., 13, 222-232.

CANDAU M., DELPON G., FIORAMONTI J., 1978a. Influence de la nature des glucides membranaires sur le développement anatomo-fonctionnel du tractus digestif du lapin. $2^{e}$ Journ. Rech. Cunic., Toulouse (communication I).

CANDAU M., BERTRAND B., FIORAMONTI J., 1978b. Variations de la digestibilité des constituants de la ration chez le lapin. $C$. $R$. Soc. Biol., 172, 554-558.

COLIN M., MAIRE C., VAISSAIRE J., RENAULT L., 1976. Etude expérimentale du remplacement, dans les aliments pour lapin, de la cellulose par les lests minéraux sable et vermiculite. Rec. Méd. vét., 152, 457-465.

DEHALLE C., 1981. Equilibre entre les apports azotés et énergétiques dans l'alimentation du lapin en croissance. Ann. Zootech., 30, 197-208.

GIDENNE T., PONCET C., 1985. Digestion chez le lapin en croissance d'une ration à taux élevé en glucides pariétaux : étude méthodologique pour le calcul de la digestibilité apparente par segment digestif. Ann. Zootech., 34, 429-446.

HYDEN S., 1955. A turbidimetric method for the determination or higher polyethylene glycols in biological materials. Ann. Roy. Agr. Coll. Sweden, 22, 139-145.

LAPLACE J. P., 1978. Le transit digestif chez les monogastriques. III. Comportement (prise de nourriture-caecotrophie), motricité et transit digestifs, et pathogénie des diarrhées chez le lapin. Ann. Zootech., 27, 225-265.

LAPLACE J. P., LEBAS F., 1977. Le transit digestif chez le lapin. VII. Influence de la finesse du broyage des constituants d'un aliment granulé. Ann. Zootech., 26, 413-420.

LEBAS F., LAPLACE J. P., 1977. Le transit digestif chez le lapin. VIII. Influence de la source de cellulose. Ann. Zootech., 26, 575-584.

MORIN N., SEROUX M., PIGANEAU P., 1979. Méthodes d'expérimentation sur le lapin. Perspectives agr., 23, 64-72.

MORISSE J. P., 1982. Taille des particules de l'aliment utilisé chez le lapin. Rec. Méd. vét., $133,635-642$.

PAIRET M., BOUYSSOU Th., AUVERGNE A., CANDAU M., RUCKEBUSCH Y., 1986. Stimulation physicochimique d'origine alimentaire et motricité digestive chez le lapin. Reprod. Nutr. Dévelop., 26, 85-95.

PARKER D. S., Mc MILLAN R. T., 1976. The determination of VFA in the caecum of conscious rabbit. Br. J. Nutr., 35, 365-374.

PICKARD D. W., STEVENS C. E., 1972. Digesta flow through the rabbit large intestine. Am. J. Physiol., 222, 1161-1166.

SCHIEMANN R., NEHRING K., HOFFMANN L., JENTSCH W., CHUDY A., 1971. Energetische Futterbewertung und Energienormen. VEB Deutscher Landwiztschaftsverlag, Berlin.

SVENDSEN P., 1972. Inhibition of caecal mobility in sheep by volatile fatty acids. Nord. Vet. Med, 24, 393-396. 
VAN SOEST D. J., 1967. Development of a comprehensive system of feed analyses and its applications to forage. J. anim. Sci., 26, 119-127.

VERNAY M., 1985. L'absorption et le devenir des acides gras volatils digestifs chez le lapin en relation avec la dualité de l'émission fécale. Thèse Doct. Etat, U.P.S. Toulouse.

VIGROUX P., 1976. Recherches sur la motricité caecale chez le cheval. Thèse Doct. $3^{\circ}$ cycle, I.N.P. Toulouse. 